\title{
FOURIER TRANSFORMS OF DINI-LIPSCHITZ FUNCTIONS ON VILENKIN GROUPS
}

\author{
M.S. YOUNIS \\ Department of Mathematics \\ Yamrouk University \\ Irbid, Jordan \\ (Received November 30, 1987 and in revised form January 17, 1991)

\begin{abstract}
In [4] we proved some theorems on the Fourier Transforms of functions satisfying conditions related to the Dini-Lipschitz conditions on the $n$-dimensional Euclidean space $R^{n}$ and the torus group $T^{n}$. In this paper we extend those theorems for functions with Fourier series on Vilenkin groups.
\end{abstract}

KEY WORDS AND PHRASES. Dini-Lipschitz Functions, Vilenkin Fourier Series. 1991 AMS SUBJECT CLASSIFICATION CODE. 42c, 43.

\section{INTRODUCTION.}

Let $f(x)$ belong to the Lebesgue space $L^{p}, 1<p \leq 2$ of functions on the real line $R$ or on the circle group $T$ with its usual norm $\|\cdot\|_{p}$. The $p$ th integral modulus of continuity $\omega_{p}(f, h)$ is defined as

$$
\omega_{p}(f, h)=\sup \|f(x+h)-f(x)\|_{p} .
$$

In [4] (Theorem 3.3) we proved that if $f(x)$ belongs to $L^{p}(R)$ such that

$$
\omega_{p}(f, h)=0\left(h^{\alpha} /(\log h)^{\gamma}, \quad o<\alpha \leq 1\right.
$$

then the Fourier Transform $\hat{f}$ belongs to $L^{\beta}(R)$ where

$$
p /(p+\alpha p-1)<\beta \leq p^{\prime}=p /(p-1), \beta>1 / \dot{\gamma} .
$$

In the present work we shall extend this result for functions on $L^{p}(G)$ where $G$ is a compact metrizable zero-dimensional Abelian group, i.e., a Vilenkin group.

\section{DEFINITIONS AND NOTATIONS.}

Here we introduce some definitions and notations that will be used later on. This is briefly done since we shall mainly follow Onneweer [1] and Quek and Yap [2] in this respect.

Let $G$ be a Vilenkin group. Then its dual $\hat{G}$ is a discrete countable torsion group. It is well known that one can introduce on $G$ a countable basic set of neighbourhoods $\left\{G_{n}\right\}$ of the identity element $\{e\}$ of $G$ such that

$$
G=G_{0} \supset G_{1}, \supset G_{2}, \ldots, \text { and }{ }_{n}^{\infty} \bigcap_{0} G_{n}=\{e\} .
$$

On the other hand, let $V_{n}$ denote the annihilator in $\hat{G}$ of the subgroup $G_{n}$ in $G$. Then it is 
known that

$$
\{e\}=V_{0} \subset V_{1}, \ldots, \subset \hat{G}, \text { and that }{ }_{n} \stackrel{0}{0}_{0} V_{n}=\hat{G} .
$$

If all $V_{n}$ are finite, the inclusion is proper. We introduce the numbers $m_{o}, m_{1}, m_{2}, \ldots, m_{k}$ such that

$$
m_{o}=1, m_{k+1}=p_{k} m_{k} ; \quad k \varepsilon N,
$$

$p_{k}$ being a prime $\geq 2$. Then every $V_{n}$ has $m_{n}$ as its measure and the quotient subgroup $V_{n} / V_{n-1}$ has $P_{n}$ for its measure.

DEFINITION 2.1. For $x \varepsilon G$, let $(n, x)$ denote the continuous character of $x$, i.e. $(n, x) \varepsilon \hat{G}$. The Fourier transform $\hat{f}(n)$ of $f(x) \varepsilon L^{p}(G)$ is defined by

$$
\hat{f}(n)=\int_{G} f(x) \overline{(n, x)} d x
$$

where $\overline{(n, x)}$ is the complex conjugate of $(n, x)$.

DEFINITION 2.2. Let $f(x) \varepsilon L^{p}(G)$. The $p$ th modulus of continuity $\omega_{p}(f, k)$ is defined by

$$
\omega_{p}(f, k)=\sup _{h \varepsilon G_{k}}\|f(x+h)-f(x)\|_{p}
$$

The Lipschitz and the Dini-Lipschitz classes in $L^{p}(G)$ are those for which $\omega_{p}(f, k)=0\left(m_{k}^{-\alpha}\right)$ and $o\left|\log m_{k}\right|^{-1}$ respectively.

DEFINITION 2.3 If every $P_{k}$ is finite as $k \rightarrow \infty$ we say that $G$ has the boundedness property (P).

3. MAIN RESULTS.

With the previous definitions and notations in hand, we now prove the analogue of Theorem 3.3 in [4]. Thus we state the following

THEOREM 3.1. Le $f(x) \varepsilon L^{p}(G), 1<p \leq 2$, such that

$$
\omega_{p}(f, k)=0\left(m_{k}^{-\alpha} /\left(\log m_{k}\right)^{\gamma}\right), \quad 0<\alpha \leq 1 .
$$

Then $\hat{f}(n) \varepsilon 1^{\beta}(\hat{G})$ for $q=P /(p-1) \geq \beta>\max (p /(p+\alpha p-1), 1 / \gamma)$.

PROOF. Since the Fourier transform of $f(x+h)-f(x)$ is given by $\hat{f}(n)(n, h)-1)$, the Hausdorff-Young theorem yields

$$
\sum_{\hat{G}}|\hat{f}(n)|^{q}|(n, h)-1|^{q} \quad<M \omega_{p}(f, k)^{q}=0\left(m_{k}^{-\alpha q} /\left(\log m_{, k}\right)^{\gamma q}\right) .
$$

The boundedness property $(P)$ for $G$ gives (see Onneweer [1], (2)).

$$
\sum_{n=m_{k}}^{m_{k}+1}|\hat{f}(n)|^{q}=0\left(m_{k}^{-\alpha q} /\left(\log m_{k}\right)^{\gamma q}\right.
$$

Applying the Holder's inequality with $\beta \leq q$ for the last estimate one arrives at

$$
\sum_{n=m_{k}}^{m_{k}+1}|f(n)|^{\beta}=0\left(m_{k}^{-\alpha \beta} /\left(\log m_{k}\right)^{\gamma \beta}\right)\left(m_{k}^{1-\beta / q}\right)
$$

and this leads to the final estimate

$$
\sum_{n \varepsilon \hat{G}}^{\infty}|\hat{f}(n)|^{\beta}=0\left(\sum_{k=0}^{\infty}\left(m_{k}^{1-a \beta-\beta+\beta / p}\right)\left(\log m_{k}^{-\gamma \beta}\right) .\right.
$$


If $1-\alpha \beta-\beta+\beta / p<0$ and $-\gamma \beta<-1$ the series is convergent since $m_{k} \geq 2^{k}$, and this proves the theorem.

REMARK 3.2. We remark here that for special choice of $\alpha, \gamma$, and $P$ like. $\alpha=1, \gamma=1$, $P=2$, the previous theorem gives special interesting cases. This is quite obvious and we shall not deal with it any further. However, the special case $P=2$ and $o<\alpha<1$ is particularly important and deserves special consideration.

4. FUNCTIONS IN $L^{2}(G)$. The origin of this section is a theorem proved in Titchmarsh ([3] Theorem 85, p. 117) for functions belonging to $\operatorname{Lip}(\alpha, 2)$ on the real line $R$. For further reference we state it as.

THEOREM 4.1. Let $f(x) \varepsilon \operatorname{Lip}(\alpha, 2)$ on $R$. Then the conditions

$$
\omega_{2}(f, h)=0\left(h^{\alpha}\right), \quad 0<\alpha<1, \quad h \rightarrow 0
$$

and

$$
\left[\int_{-\infty}^{-X}+\int_{X}^{\infty}\right]|\hat{f}|^{2} \mathrm{du}=0\left(X^{-2 \alpha}\right) \text {, as } X \rightarrow \infty
$$

are equivalent.

This theorem was studied rather extensively in [5] and [6] for functions in $L^{2}\left(R^{2}\right)$, and $L^{2}\left(T^{2}\right)$ respectively, where several conditions of the order of magnitude for the Fourier transforms $\hat{f}$ of $f$ proved to be equivalent to one another.

In [4] (Theorems 5.1, 5.2) we proved an analogue of Theorem 4.1 for the Dini-Lipschitz functions in $L^{2}(R)$. In this section we shall prove Theorem 5.2 in [4] for functions in $L^{2}(G)$.

THEOREM 4.2. Let $f(x)$ belong to $L^{2}(G)$. Then the conditions

$$
\omega_{2}(f, k)=0\left(h^{\alpha} /(\log h)^{\gamma}\right), \quad h \varepsilon G_{k}
$$

and

$$
\sum_{n=m_{k}}^{\infty}|\hat{f}(n)|^{2}=0\left(m_{k}^{-2 \alpha} /\left(\log \left(m_{k}^{2}\right)^{\gamma}\right)\right.
$$

are equivalent. Here $h=m_{k}^{-1}$.

PROOF. That the first implication is true follows from Theorem 3.1 where it is proved that

$$
\sum_{n=m_{k}}^{\infty}|\hat{f}(n)|^{q}=0\left(\omega_{p}(f, k)\right)^{q}
$$

Taking $p=q=2$ and substituting for $h=m_{k}^{-1}$ we obtain (4.2). We also hint that an argument based on the Parseval's identity similar to that of Titchmarsh's leads independently to the same result. To prove the converse let (4.2) hold. Then

$$
\sum_{n=m_{k}}^{m_{k+1}-1}|\hat{f}(n)|^{2}=0\left(m_{k}^{-2 \alpha} /\left(\log m_{k}^{2 \gamma}-0\left(m_{k+1}^{-2 \alpha} /\left(\log m_{k+1}\right)^{2 \gamma}\right)\right.\right.
$$

Since $G$ has the boundedness property $(P)$; hence every $P_{k}=m_{k+1} / m_{k}$ is finite for all $k \varepsilon N$, the same is true of $\log P_{k}$. Thus the right hand sides of (4.2) and (4.3) are the same. This applies to estimates of the form

$$
\sum_{n=m_{k}}^{m_{k+1}-1}|\widehat{f}(n)|^{2}|(n, h)-1|^{2}
$$


To sum up, by the Parseval's identity one obtains

$$
\begin{aligned}
\int_{G}|f(x+h)-f(x)|^{2} d x & =\sum_{\hat{G}}|\hat{f}(n)|^{2}|(n, h)-1|^{2} \\
& =\sum_{m_{k}}^{\infty}+\sum_{0}^{m_{k}}=0\left|m_{k}^{-2 \alpha} /\left(\log m_{k}\right)^{2 \gamma}\right|
\end{aligned}
$$

This is equivalent to (4.1) upon substituting for $h=m_{k}^{-1}, h \varepsilon G_{k}$ and the proof is complete.

REMARK 4.2. We conclude by indicating that Theorem 5.1 in [4] is true for Vilenkin Fourier series, since, it can be deduced as a special case of Theorem 4.1. We also add that for $0<\alpha<1$, Theorems 3.1 and 4.1 of the present paper can be proved for higher differences of $f(x) \varepsilon L^{p}(G)$. The statements and the proofs are almost straightforward and will not be given.

ACKNOWLEDGEMENT: This research was supported by a grant from Yarmouk University.

\section{REFERENCES}

1. ONNEWEER, C.W., Absolute Convergence of Fourier Series on Certain Groups. II, Duke, Math. Journal, Vol. 41 (1974), 679-688.

2. QUEK, T.S. and YAP, L.Y.H., Absolute Convergence of Vilenkin-Fourier. Series, L. Math. Analysis and Appl. Vol. 74 (1980) 1-14.

3. TITCHMARSH, E.C., Theory of Fourier Integrals, 2nd Ed. Oxford Univ. Press, 1948.

4. YOUNIS, M.S., Fourier Transforms of Dini-Lipschitz Functions, Internat. J Math \& Math. Sci. Vol. 9 No. 2 (1986) 301-312.

5. YOUNIS, M.S., Fourier Transforms in $L^{p}$ spaces, M. Phil. Thesis, Chelsea Coll., London, 1970.

6. YOUNIS, M.S., Fourier Transforms of Lipschitz Functions on compact Groups, Ph.D. Thesis, McMaster Univ., Hamilton, Ontario, Canada, 1974. 


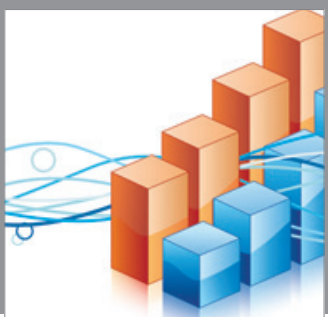

Advances in

Operations Research

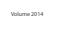

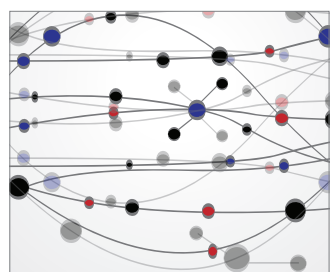

\section{The Scientific} World Journal
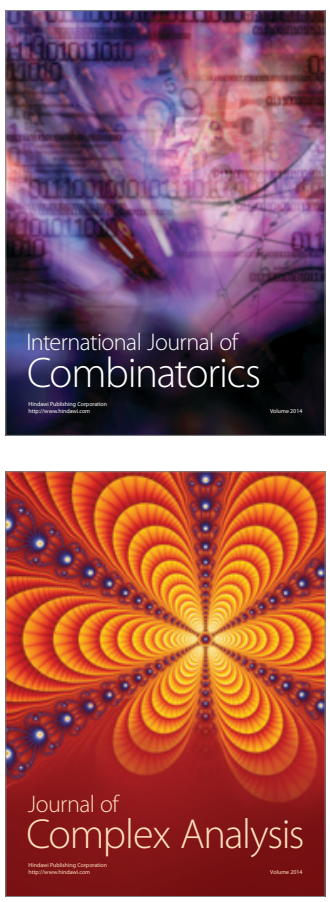

International Journal of

Mathematics and

Mathematical

Sciences
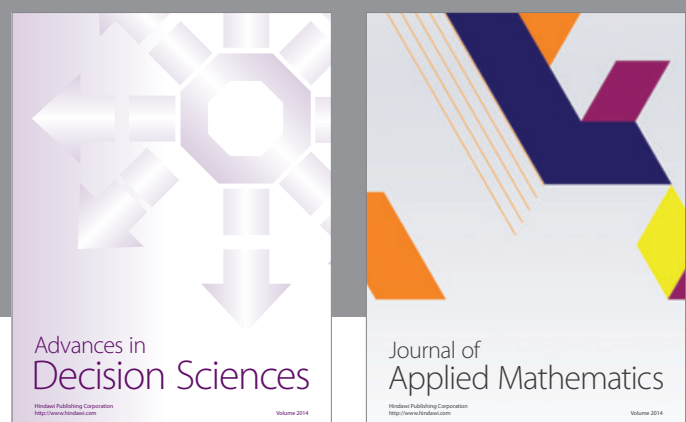

Journal of

Applied Mathematics
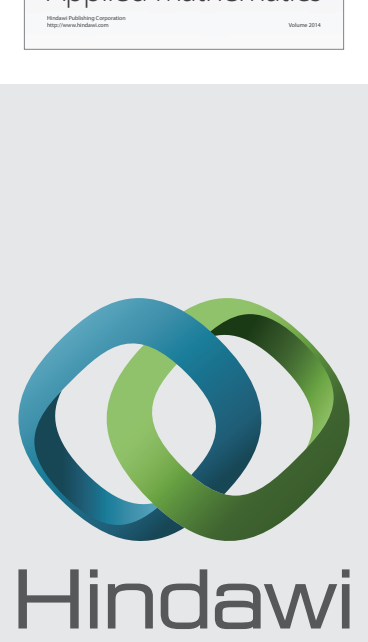

Submit your manuscripts at http://www.hindawi.com
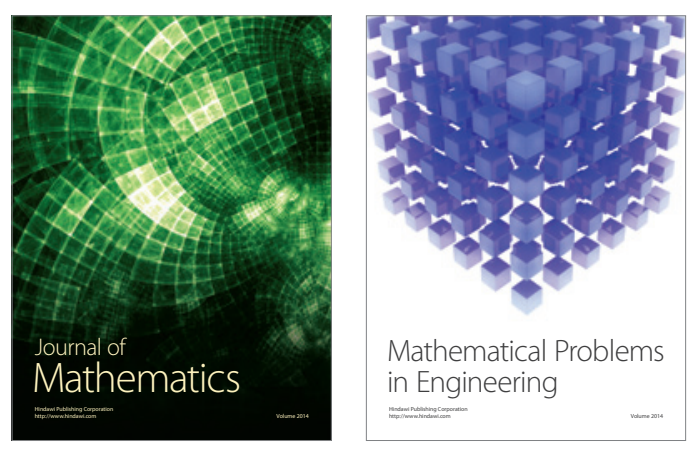

Mathematical Problems in Engineering
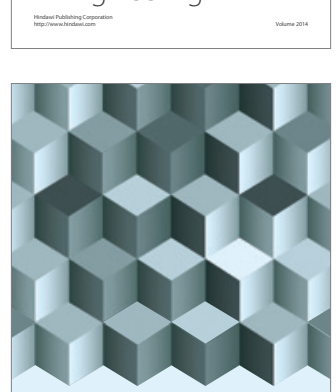

Journal of

Function Spaces
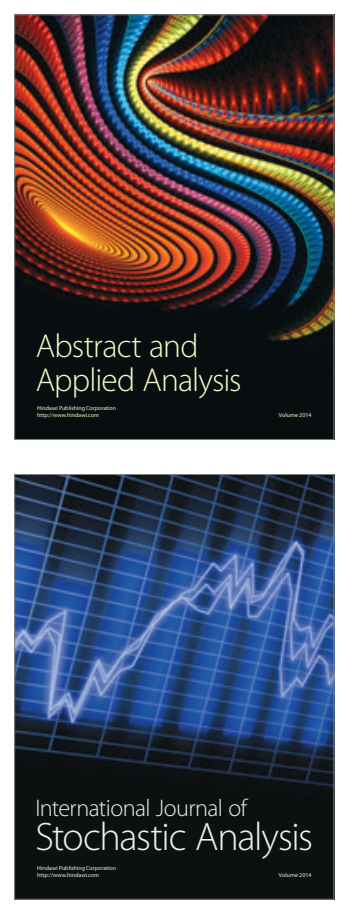

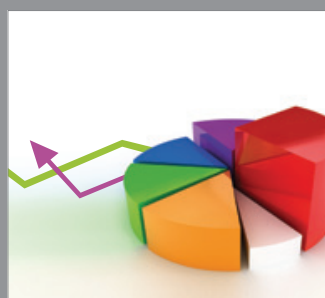

ournal of

Probability and Statistics

Promensencen
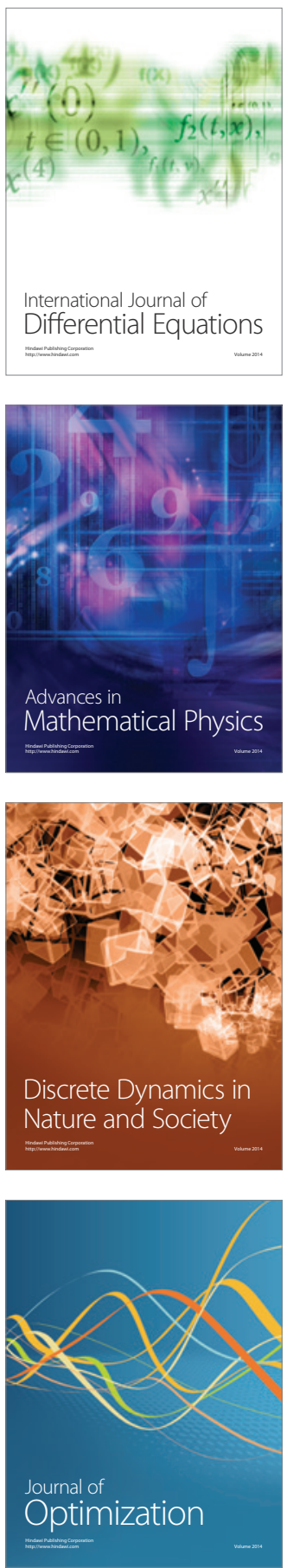